\title{
The cryoloop: an adaptable reversible cooling deactivation method for behavioral or electrophysiological assessment of neural function
}

\author{
Stephen G. Lomber ${ }^{\mathrm{a}, *}$, Bertram R. Payne ${ }^{\mathrm{a}}$, James A. Horel ${ }^{\mathrm{b}}$ \\ a Laboratory for Visual Perception and Cognition, Department of Anatomy and Neurobiology, Boston University School of Medicine, \\ 700 Albany Street, Boston, MA 02118, USA \\ ${ }^{\mathrm{b}}$ Department of Anatomy and Cell Biology, State University of New York, Health Science Center at Syracuse, 766 Irving Avenue, Syracuse, \\ NY 13210, USA
}

\begin{abstract}
We describe a very adaptable reversible inactivation technique for the behavioral or electrophysiological analysis of neural circuits. The cryoloop device can be permanently implanted or topically applied in an acute preparation to apply cold to discrete surface regions of the central nervous system (e.g. cerebral cortex or midbrain). The cryoloop consists of a custom shaped, stainless steel, hypodermic tubing and cooling is effected by passing chilled methanol through the lumen of the tubing. Cryoloop temperature is monitored by a microthermocouple attached to the union of the loop, and can be maintained within $\pm 1^{\circ} \mathrm{C}$ of a desired temperature. In chronic preparations, implanted cryoloops have been maintained in cats and monkeys for periods in excess of 2 years. After this period there are no structural, metabolic of functional changes in the deactivated tissue, and full reversibility of cooling-induced effects is maintained. Operation of multiple cryoprobes provides great flexibility of experimental protocols, permits double and triple functional dissociations to be made, and strengthens experimental design considerably. (C) 1999 Elsevier Science B.V. All rights reserved.
\end{abstract}

Keywords: Cerebral cortex; Superior colliculus; Perception; Cognition; Modeling

\section{Background}

Studies of the contributions made by cortical areas to network interactions and cerebral function require the deactivation of individual areas. For many investigators the lesion technique is the method of choice to silence a region of cortex because the limits of the lesion can be defined with a reasonable level of accuracy. However, there are drawbacks to this method: (1) typically lesions can only be defined post mortem; (2) it is not possible to reinstate the damaged region in the network to obtain control measures that bracket the lesion-induced effects; 3) comparisons must be made between animals and internal double dissociations are not possible; and (4) the mature CNS is plastic and connections can be activated, strengthened or modified following lesions

\footnotetext{
* Corresponding author. Tel.: +1617 6384188; fax: + 1617 6384102; e-mail: slomber@bu.edu
}

(Yamasaki and Wurtz, 1991; Lomber et al., 1996b; Payne et al., 1996b). Therefore, it is most desirable to use reversible deactivation techniques to investigate functional roles of, and interactions between, components in the cerebral network and obviate these drawbacks.

The cooling method to reversibly deactivate neural tissue is important and has several major advantages over the traditional lesion technique: (1) Limited regions of cortex can be selectively and reversibly deactivated in a controlled and reproducible way, and baseline and experimental measurements can be made virtually concurrently (within minutes). (2) Repeated coolings over several months or years produce stable, reversible defects, with no evidence of attenuation, neural compensations or major variations. (3) Repeated cooling induces neither local nor distant degenerations that might compromise conclusions and do not induce the types of neural compensations triggered by lesions. 
(4) Compared to ablation studies, many fewer animals are needed because sensitive within animal comparisons and double, or even triple, dissociations are possible, permitting large volumes of high quality data to be acquired from each animal. (5) Finally, because the major effect of the cooling is to block synaptic transmission, activity in fibers of passage is not compromised.

A number of strategies have been adopted to cool and deactivate neural tissue. Cryotips are popular for cooling deep brain structures, where the site of cooling is desired to be as localized as possible. Cryotips are concentric tubes through which a coolant is passed down the inside shaft to localize the cooling to the tip of the shaft (Dondey et al., 1962). Additional heating elements have also been added to the outside shaft to prevent the unintentional inactivation of other, more superficial structures (Skinner and Lindsley, 1968; Zhang et al. 1986; Campeau and Davis, 1990). However, penetration of overlying structures inevitably results in some damage and they are not very useful for cortical deactivations. For surface structures, such as cerebral cortex, the most frequently adopted method employs the Peltier principle and thermoelectric plates. These have been used in electrophysiological investigations, where the cooling of large, relatively smooth, cortical areas has been successfully carried out (Schiller and Malpeli, 1977; Sherk, 1978; Sandell and Schiller, 1982; Girard and Bullier, 1989; Girard et al., 1991a,b, 1992; Michalski et al., 1993, 1994). In behavioral studies the plates are positioned outside the dura, a structure which tends to insulate the underlying brain from the cooling effect (Fuster and Alexander, 1970; Fuster and Bauer, 1974; Goldman and Alexander, 1977; Alexander and Goldman, 1978; Shindy et al., 1994). Moreover, the plates rarely conform exactly to the shape of the underlying neural surface to be cooled, and the animal's head must be immobilized. To our knowledge, cooling plates have not been successfully introduced into sulci.

The cryoloop technique for cooling was originally described by Salsbury and Horel (1983) and adopted for epidural use in behaving monkeys without the need for head restraint because the cryoloops were chronically implanted (Horel, 1991). More recently, the technique has been advanced and chronically implanted subdurally in cats by Lomber and Payne. Moreover, techniques have been developed to introduce cryoloops successfully into sulci. With these recent advances, broader animal use, and implementation of the technique in a growing number of laboratories it seemed appropriate to provide a thorough description of assembly, implantation procedures, maintenance and use of the cryoloop. The cryoloop method can be applied to assess cortical operations using, with equal success, behavioral or electrophysiological assays of neural operations (Horel, 1984, 1992, 1996; Horel et al. 1984, 1987; Payne et al., 1991, 1996a; Lomber et al., 1994, 1996a,b;
Lomber and Payne, 1996; Girard et al. 1997; Hupé et al., 1998).

\section{The cryoloop}

\subsection{Parts and assembly}

(1) The cryoloop is manufactured from straight 23 gauge $(0.635 \mathrm{~mm}$ O.D. $\times 0.33 \mathrm{~mm}$ I.D.) hypodermic stainless steel tubing. Thinner walled tubing may be used but it is more difficult to fashion without crimping or denting. The tubing is cut with a high-speed, handheld hobby drill (e.g. Dremel tool). The tubing is then shaped with round, needle-nosed pliers into a simple loop, or more complex loop with one or more secondary side loops, and it is shaped to conform to the surface of the structure to be deactivated. For this step we use fixed brains or rubber replicas as forms to shape the cryoloops. A single loop can be constructed to deactivate between $\sim 10$ and $100 \mathrm{~mm}^{3}$ of cortex. Deactivations of $>100 \mathrm{~mm}^{3}$ can be achieved by deactivating adjacently positioned cryoprobes.

(2) A microthermocouple is constructed and attached to the cryoloop. The microthermocouple is manufactured by simply twisting together bared $30 \mathrm{AWG}$ gauge $(0.254 \mathrm{~mm})$ Teflon insulated copper and constantan wire and closely trimming the tips. The copper/constantan duplex has both high resolution and fast response time in the operating range of $0-40^{\circ} \mathrm{C}$.

(3) The microthermocouple is soldered to the union of the cryoloop with silver solder after flux has been applied to the tubing. Afterwards, excess toxic flux is rinsed away.

(4) The paired inlet and outlet tubes of the cryloop, the microthermocouple wire, and the solder embedded microthermocouple are encased in 16 gauge heat-shrink Teflon tubing (expanded diameter of $1.7 \mathrm{~mm}$; Small Parts Inc., Miami Lakes, FL; Part \# SMT-16-12) leaving the cryoloop exposed.

(5) The inlet and outlet tubes and microthermocouple wire are led through a stainless steel, outside-threaded, cylinder and secured together with dental acrylic (e.g. Kerr $^{\circledR}$ Fastcure).

(6) The thermocouple wires exit through a small hole and are attached to an Omega Engineering ${ }^{\circledR}$ subminiature connector ( \# SMP-T).

(7) An inside-threaded tube cut into short lengths and sealed at one end with dental acrylic is screwed into place as a protective cap for the inlet and outlet tubes. A set-screw is employed on monkey cryoprobes to prevent the monkey from manually manipulating the protective cap free.

Finally, the patency of the cryoloop tubing is tested for methanol flow, functioning of the microthermocou- 

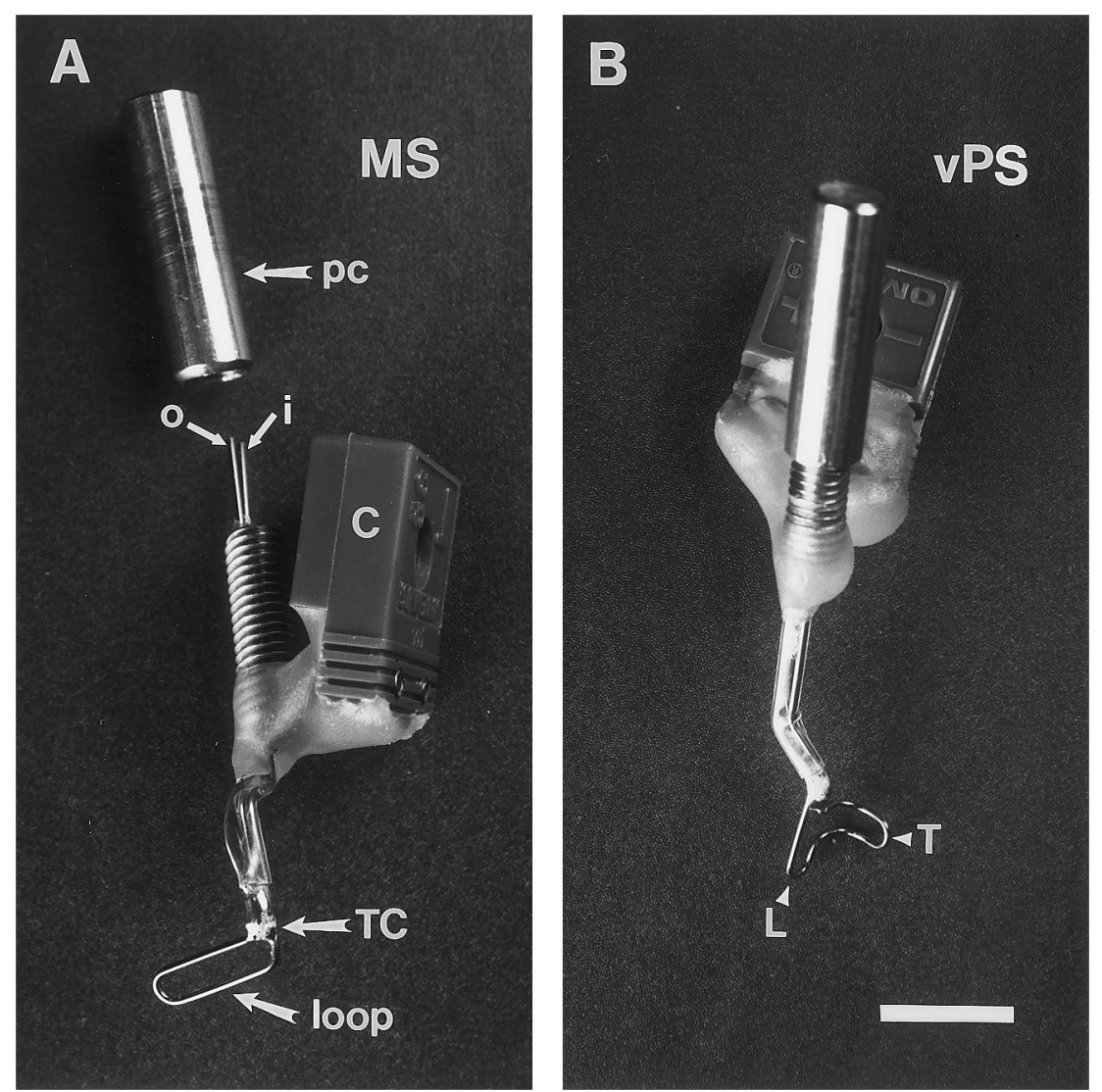

Fig. 1. Individual cortical cryoloops constructed to be placed: (A) in the left middle suprasylvian (MS) sulcus, or (B) over the right ventral-posterior suprasylvian (vPS) and fusiform gyri of the cat. (A) Left MS cryoloop. Protective cap (pc) has been removed to expose the ends of the inflow (i) and outflow (o) tubes. Tubes descend through the threaded post and the heat-shrink Teflon tubing to the actual loop which is exposed to the cortical surface. The microthermocouple (TC) is attached to the union of the cryoloop. Its wires ascend through the heat-shrink Teflon tubing to the connector (C) affixed to the dental acrylic. (B) Right vPS cryoloop. Similar overall construction. Note that the loop is shaped for contact with the lateral occipital surface (L; vPS cortex) and the tentorial surface (T; fusiform gyrus). Here, the protective cap is in place over the inflow and outflow tubes. Scale bar, $10 \mathrm{~mm}$.

ple is verified, and the construction of the standard cryoloop blank is complete. Two assembled cryoloops are shown in Fig. 1.

\subsection{Surgical implantation}

Every region of the brain to be deactivated presents its own unique surgical challenges. The general procedure described below is readily adaptable, with some forethought, to the desired region of the brain to be cooled. At present, six cryoloops have been implanted in a single cat and ten into a single monkey. The larger monkey brain and skull can accommodate more cryoloops, as can those of the cat with a reduction in the size of exposed portions of the cryoloops. However, the major factor limiting the number of cryoprobes that can be implanted is the duration of the surgery. At present, the implantation of six surface cryloops in cats requires about $10 \mathrm{~h}$ in surgery. More time is required when sulci first need to be opened. There are clear advantages to implanting more cryoprobes, but they should be counterweighed by the need for two surgical procedures. If two surgeries are contemplated, it is most practical to implant probes in contact with one hemisphere in one surgery, and to implant cryoprobes in contact with the opposite hemisphere in a second surgical procedure. Naturally, with all invasive surgery, sterile technique is essential, and all procedures should be approved by the institution and conform with local and national guidelines.

(1) Cryoloops are sterilized with ethylene oxide because autoclaving is destructive to both the cryoloops and microthermocouples.

(2) With the animal under general, surgical plane anesthesia, the skull is exposed and a craniotomy is made over the desired region. The craniotomy should be large enough to permit mechanical manipulation of the brain and manipulation of the cryoloops into place. However, sufficient skull should remain in place to permit firm anchoring of the cryoloop.

(3a) For surface positions, placement is straightforward. For sulcal positions, it is essential to dissect the arachnoid mater bridging across the sulcal orifice tak- 
ing great care not to compromise the vascular supply. Fine Science Tools $^{\circledR}$ etched tungsten microneedles (\# 10130-20) and curved microforceps are particularly useful for dissecting the arachnoid mater. Once the more difficult and extensive surface arachnoid mater has been dissected it is straightforward to sever the small number of bridging strands connecting the two banks within the sulcus. It is essential to pay attention to vessels which bridge across the sulcus. These can be a hazard, and in some cases it may be impossible to navigate the cryoloop around them. While we have not encountered difficulties with vasculature, we anticipate that at some point in the future we will encounter an arterial supply or venous drainage pattern which prevents implanting a cryoloop in a sulcus on these grounds. Unfortunately, it is impossible prior to opening the dura to know whether a given surgery is unfeasible. Thus, it is worthwhile implanting cryoprobes early in an experiment, soon after an animal is accustomed to the behavioral apparatus, rather than training the animal on a large number of tasks and then implanting the cryprobe.

(3b) Good illumination and a view parallel to the sulcal walls are essential. A motorized-zoom and -focus surgical microscope is highly recommended because both hands are occupied gently pulling the two banks apart and opening the sulcus. The motorized focus permits focusing to successive depths in the sulcus as it is opened, and it is essential for clear progress. Dental cotton rolls are readily available, and have a good shape and texture for applying to the cortical surface for gently separating the sulcal banks. We have enjoyed success opening the middle suprasylvian sulcus of cats (Fig. 2A; Lomber et al., 1994, 1996b; Payne et al., 1996a) and superior temporal sulcus of monkeys (Hupé et al., 1998). For deep structures, such as the superior colliculus, one hemisphere is elevated after a relieving cut is made in the splenium of the corpus callosum (Fig. 2B; Lomber and Payne, 1996; Payne et al., 1996a). The superior colliculus is readily visible as a vascularized, cream-colored dome when viewed with fiber optic light. It lies immediately in front of the tentorium cerebelli. Bright illumination is essential.

(4) One of the standard probes most suitable in size, shape and position of inlet and outlet tubes and thermocouple connector is selected, and the concordance of the loop with the shape of the brain is tested. Final adjustments are made to maximize concordance between cryoloop and brain, and eliminate interference between external elements of other cryoloops.

(5) The skull is cleaned and holes are drilled with a hand drill to prevent scorching of the bone. Silver sputtered $^{1}$ stainless steel screws (Small Parts Inc., Mi-

\footnotetext{
${ }^{1}$ Screws can be conveniently sputtered using a sputterer suitable for preparing replicas for a scanning electron microscope.
}

ami, FL; Part \# MPX-080-2P) are self-tapped into the drilled holes to serve as anchors for the cryoloop assembly.

(6a) The cryoloop assembly is positioned in place and secured to the screws with dental acrylic that contains $1 \%$ silver sulfate by weight. The silver on the screws and in the acrylic retards infections, which can lead to deterioration of the bone and be hazardous to overall health of the animal. Bone deterioration can result in instability and, ultimately, in detachment of the cryoprobe.
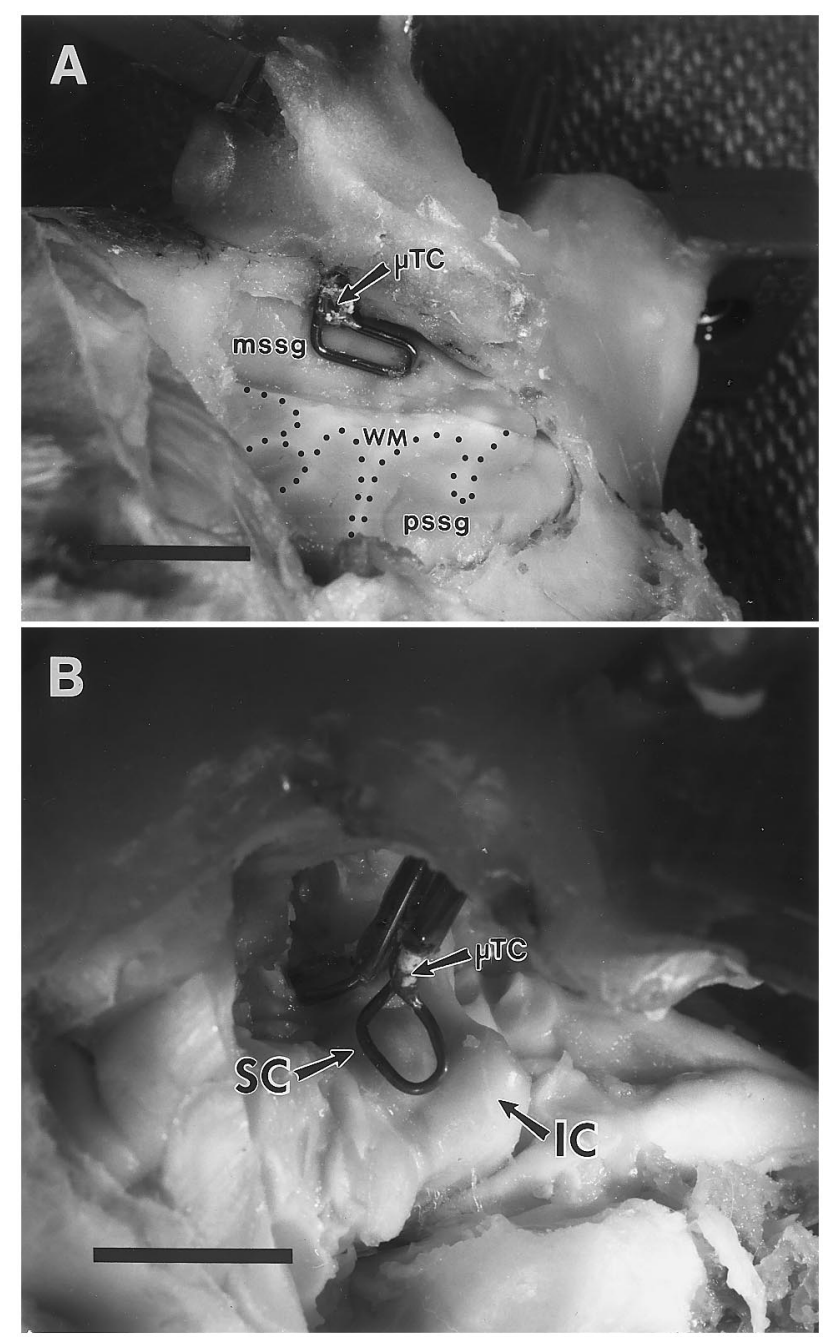

Fig. 2. Cooling probes in contact with posterior-middle suprasylvian (pMS) cortex and the superior colliculus (SC) of the cat. (A) Position of a cooling probe in the posterior middle suprasylvian sulcus. Probe is resting against the middle suprasylvian gyrus (mssg) which forms the medial bank of the MS sulcus. Cortex lateral to the probe was removed following tissue fixation to reveal the probe. Left is anterior. $\mathrm{WM}$, white matter; pssg, posterior suprasylvian gyrus; UTC, microthermocouple. Scale bar, $10 \mathrm{~mm}$. (B) Photograph to show the position of the cooling probe in contact with the superior colliculi. Overlying cortex was removed to reveal the underlying thalamus, midbrain, and probe position. Left is anterior. IC, inferior colliculus. Scale bar, $10 \mathrm{~mm}$. Reproduced from Lomber and Payne (1996) with permission. 
(6b) The most convenient way to hold the cryoprobe in place during fixation to the skull is manually using visual feedback to control exact position. Even with the steadiest of hands it is essential to support the hand to minimize movements. The use of slow curing acrylic should be avoided as the probe will then need to be manually held in place for a long period of time.

(6c) In applying the acrylic neither air nor fluid pockets are tolerated between skull and acrylic because they can harbor infectious microbes. Therefore, the initial layer of dental acrylic applied to the screws and skull has a low viscosity and is allowed to run freely over all surfaces. Subsequent layers are built up using a higher viscosity slurry of acrylic.

(7) Dural margins are sutured, as needed, absorbable gelatin film (Gelfilm ${ }^{\circledR}$, The Upjohn Company, Kalamazoo, MI; NDC 0009-0297-01) is applied if necessary, and bone pieces replaced. Large bone pieces have at least one anchoring screw to hold them in place. Any remaining gaps between skull, bone pieces and cryoprobes are filled with absorbable gelatin foam $\left(\right.$ Gelfoam $^{\circledR}$, The Upjohn Company, Kalamazoo, MI; NDC 0009-0315-03), which prevent contact between acrylic and brain. Eventually, both Gelfilm and Gelfoam are absorbed and replaced by connective tissue or new bone. In many instances for cryoloops that have been in place for 1 year or more, the margins of the defect in the calvarium are completely ossified and barely detectable (Fig. 3). Upon closure of the cranial vault, additional acrylic is applied to secure the cryoprobe, and it is shaped to a smooth, convex contour.

(8) Finally, the skin margins are drawn up to the edge of the acrylic or are sutured together.

\subsection{Post-implantation care}

Depending upon the species, specific problems can develop with permanently implanted cooling probes, as with other implants. In cats, cryoloops have been maintained in place with little or no maintenance to the implant for 3 years. The margins of the implant are cleaned periodically with Nolvasan and treated with Panalog ointment (Nystatin-Neomycin Sulfatethiostrepton-Triamcinolone Acetonide). With these precautions neither infections nor skull deterioration are experienced. Suspected infections can be treated with Flo-Cillin (Penicillin G Benzathine and Penicillin G Procaine) administered systemically for 7-14 days. Monkeys, however, are a greater challenge as they appear to be more susceptible to infection and the skull beneath the implants tends to deteriorate over time. Careful attention to surgical precautions can greatly forestall these types of problems. However, when implants remain in place for long periods ( $>1$ year) infections or skull deterioration seem to be inevitable at

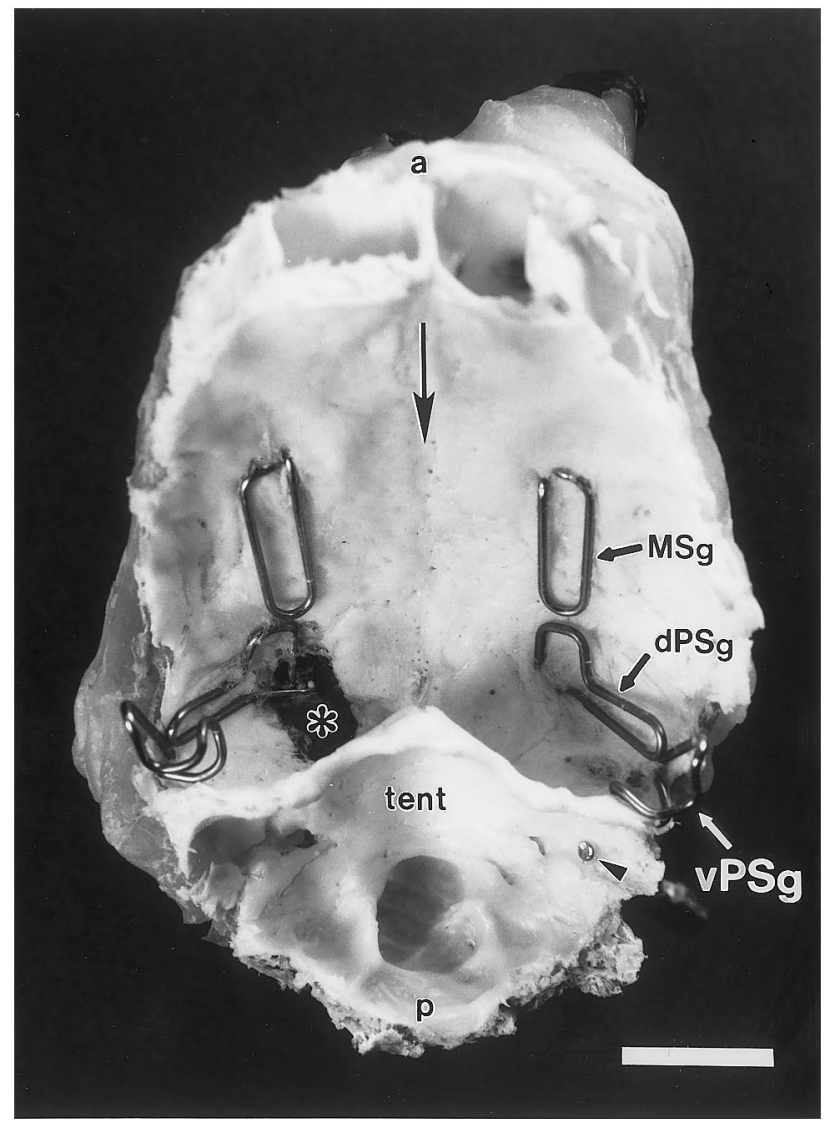

Fig. 3. Ventral view (from below) of a cat skull calvarium post-mortum to show three pairs of bilateral cryoloops. Three pairs of bilateral cryoloops had been placed over the cerebral cortex and permanently implanted approximately 30 months prior to sacrifice. MSg) Cryoloop implanted over the middle suprasylvian gyrus (cortical area 7; Olson and Lawler, 1987). dPSg) Cryoloop placed over the dorsal-posterior suprasylvian gyrus and PS-MS junction (cortical area 21a; Tusa and Palmer, 1980). vPSg) Cryoloop placed over the ventral-posterior suprasylvian gyrus which wraps around onto the tentorial surface over the fusiform gyrus (cortical area 20a and 20b; Tusa and Palmer, 1980). Note complete repair of bone above the cryoloops. Asterisk indicates site of post-mortem bone removal to show cryoloop inlet/ outlet. a, anterior; p, posterior; tent, tentorium; and arrow indicates bone impression of the superior sagittal sinus on the midline. Arrowhead indicates anchoring screw in occipital bone. Scale bar, $10 \mathrm{~mm}$.

some point in time. To prevent or treat possible infections, the margin of the acrylic is scrubbed with Betadine, inflammations flushed with Betadine, and tissue treated with Bacitracin zinc ointment or any common triple antibiotic (Polymixin, B-Bacitracin, Neomycin) ointment. If infection is suspected, the monkey can be treated systemically with Chloramphemicol or like antibiotics for 7-14 days.

\subsection{Operation}

The cryoloop is cooled by passing chilled methanol (histological grade) through the lumen of the tubing. The cooling circuit is shown in Fig. 4. 
(1) The inlet and outlet of the cryoloop fit snugly into $0.5 \mathrm{~mm}$ I.D Teflon tubing (Varian Associates, Walnut Creek, CA; Part \# R0000200332), and no special connector is required because the fit is tight enough to withstand the pressures generated by the methanol pump.

(2) Room temperature methanol is drawn from a loosely stoppered reservoir by a synchronous rotating and reciprocating piston pump (Fluid Metering Inc., Oyster Bay, NY; Model \# QSY-Q-1-CSC with \# R479 low flow isolation adapter) through $3.0 \mathrm{~mm}$ O.D. $\times 1.5 \mathrm{~mm}$ I.D. Teflon tubing (Varian Associates, Walnut Creek, CA; Part \# R000020032). It exits the pump through $2 \mathrm{~m}$ of $1.5 \mathrm{~mm}$ O.D. $\times 0.5 \mathrm{~mm}$ I.D. Teflon tubing (Part \# R000020033), which is coiled and immersed in a bath of methanol and dry-ice pellets and contained within a Nalgene ${ }^{\circledR}$ Dewar vacuum flask. The methanol/dry-ice mixture cools the flowing methanol in the tube as low as $-75^{\circ} \mathrm{C}$. The chilled methanol is then pumped onto and through the cryoloop that is in contact with the brain and, finally, back to the reservoir. The coldest cryoloop temperatures are reached by keeping the distance between the methanol/ dry ice bath and the cryoloop to a minimum. Distances

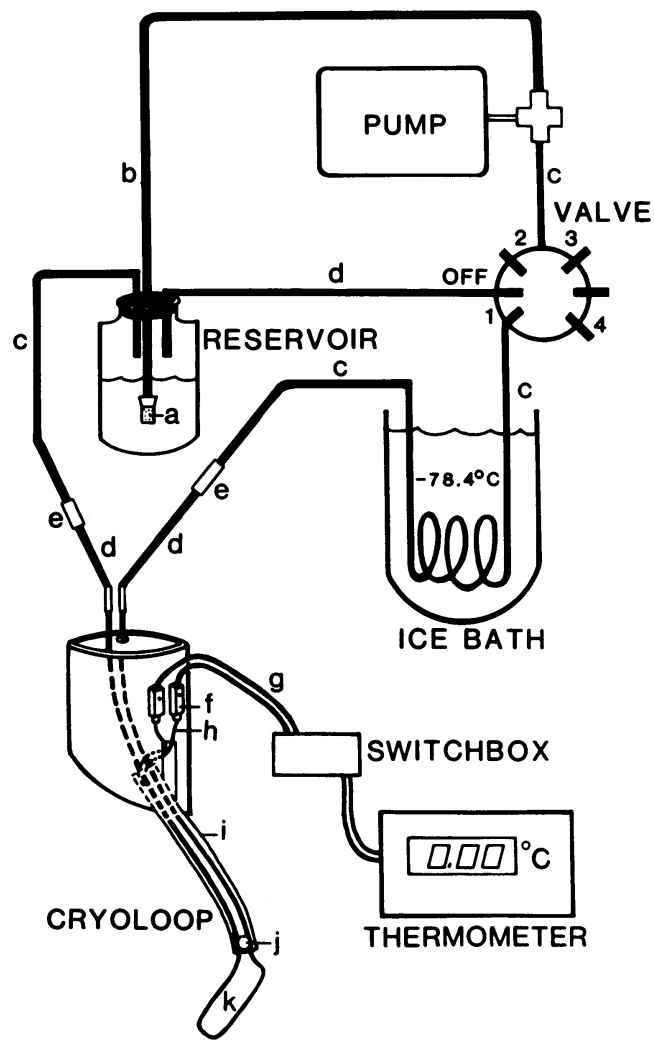

Fig. 4. The cryoloop cooling system. (a) Pump inlet and filter; (b) 1.5 $\mathrm{mm}$ i.d. Teflon tubing; (c) $0.8 \mathrm{~mm}$ i.d. Teflon tubing; (d) $0.5 \mathrm{~mm}$ i.d. Teflon tubing; (e) tubing connector; (f) thermocouple connector; ( $g$ and h) thermocouple wire; (i) heat-shrink Teflon tubing sheath; (j) thermocouple; (k) cryoloop of 23 gauge stainless steel hypodermic tubing. Reproduced from Salsbury and Horel (1983) with permission. greater than $1 \mathrm{~m}$ should be avoided unless the delivery tube is insulated.

(3) The temperature of the cryoloop is monitored by the copper/constantan microthemocouple which is connected to a digital thermometer. We have had great success with both Sensortek (Clifton, NJ: Model \# BAT 9 or 12) and Omega Engineering (Stamford, CT; Model \# HH25TC) digital thermometers.

Cryoloop temperature is influenced by the temperature of the methanol when it reaches the cryoprobe, the methanol flow rate, and the ability of the brain to counteract the cooling effect. Thus, visual monitoring of cryoprobe temperature and manual control of flow rate is sufficient to maintain temperature at a desired level $\pm 1^{\circ} \mathrm{C}$ for any length of time.

(4) Cooling deactivation is terminated by turning the pump off. It is not necessary, or advised, to pump warm methanol through the cryoloop. Brain temperature and cryoprobe temperature return to normal levels within minutes, because of the constant infusion of warm blood into the previously cooled tissue. Be aware that at termination of cooling any frozen condensate on the delivery tube thaws and may drip onto the equipment or animal and startle or irritate it.

\subsection{System maintenance}

Overall, the system is easy to operate and maintain. Useful precautions to take are to check tubing and connectors for leaks, and to change the reservoir methanol on a regular basis. About once per week is adequate when the system is in high use. This precaution keeps the methanol clean of small particles which could possible block the cryoloop. Usually, application of high pressure nitrogen is sufficient to expel any blockage that might occur.

An additional preventative measure is to ensure that animals are housed in a 'cryoprobe friendly' environment. For cats, individual caging should be avoided as the cats often rub exposed parts of the cryoprobe assembly on the sides of the cage or wire grating. Group housing in large cages obviates this problem. If individual housing is necessary, as with monkeys, the wire mesh of the cage should be as wide as possible to minimize the risk of probe becoming caught in a mesh hole.

\section{Structural and functional integrity of cortex}

We have verified that the surgical procedures to implant the cryoporobes, their presence in contact with the cortex, or their operation disturbs neither the normal structural nor functional integrity of cortex. In every instance cell and myelin stains are rich, and the cyto- and myelo-architecture of the region are charac- 


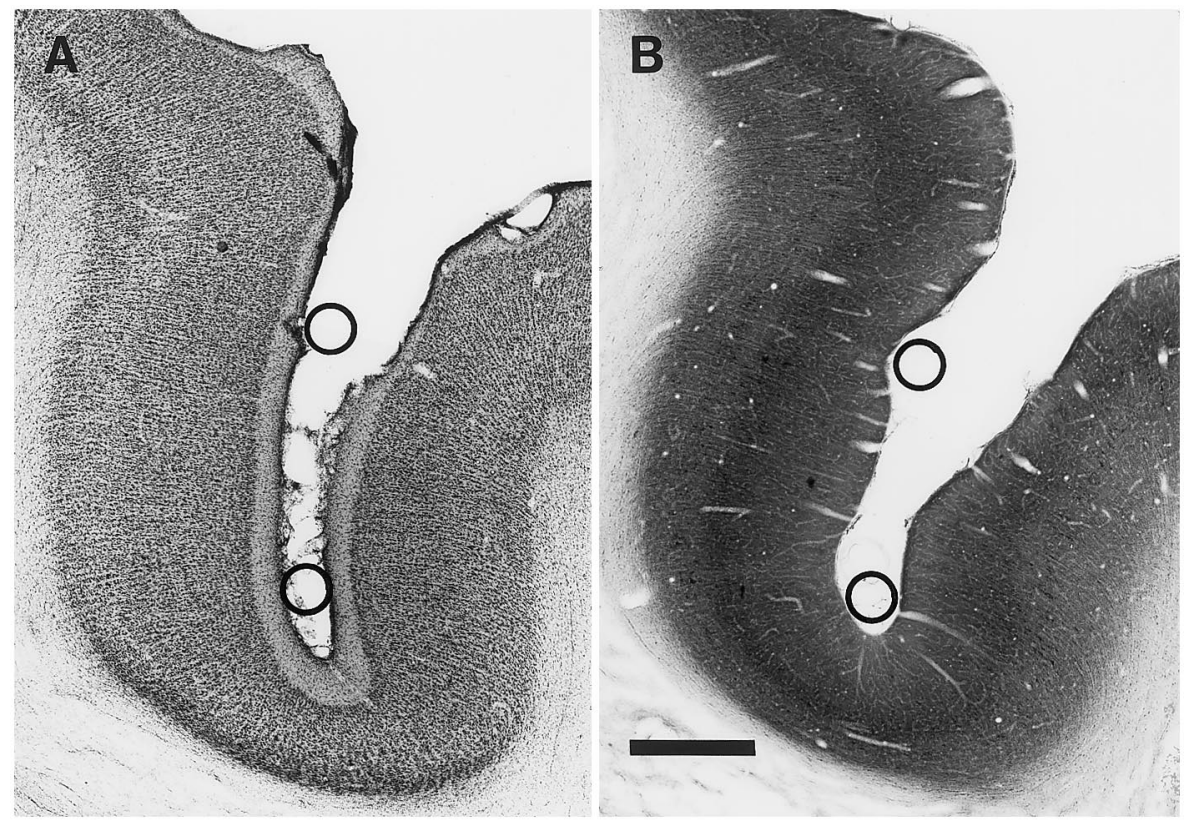

Fig. 5. (A) Photomicrograph of a cresyl violet-stained coronal section to show the microstructure of the medial (left) and lateral (right) banks of the middle suprasylvian sulcus of the cat. The impressions formed on the cortical surface by the cooling probe are evident. Overall, distortion is minimal, and cortical layers are readily identifiable. Circles represent the size and potion of the cooling probe in cross section. No mechanical or cooling damage is evident. (B) Nearby section stained for cytochrome oxidase activity. Small depressions in the cortical surface indicate position of the cooling probe loop (black circles). Left is medial. Scale bar, $2 \mathrm{~mm}$.

teristic of the region with no signs of pathology, as might be revealed by a marked pale staining of neurons or gliosis (Fig. 5A), or light staining of cytochrome oxidase (Fig. 3B). Cytochrome oxidase is a sensitive marker for pathology, abnormal functioning cortex, and for demarcating lesions (Pasternak and Merrigan, 1994). The only evidence of the presence of probes in contact with cortex is small impressions on the cortical surface where the cortex abutted the cryoprobe (Fig. 5B).

Electrophysiological procedures verify normal neuronal responses in the vicinity of the cryoloop and show the effects of the cooling on neurons. For example, Fig. 6 shows three visually responsive neurons activated by a a visual stimulus swept to and fro across the neurons' aggregate receptive field in a cat which experienced more than 100 coolings over a 6 month period. A visual stimulus moved to and fro across the neurons' aggregate receptive field reliably activated the neurons while the probe and adjacent brain region were warm, but when the chilled methanol was circulated through the cryoloop (ON) the temperature of the cryoloop fell rapidly and the neurons were silenced. They remained silent for the duration of the cooling. When the chilled methanol circulation was halted (OFF) the temperature of the cryoprobe climbed in response to the active warming effects of fresh blood perfusing the adjacent cortex. As the cortex warms, neuronal activity returns, to reveal the reversibility of the cooling effect. At this point it is important to note that the temperature registered at the cryoloop microthermocouple lags cortical temperature by $2-3 \mathrm{~min}$.

Fig. 7 shows conventional histograms of two simultaneously assayed neurons in the middle suprasylvian cortex of cat. Histological verification showed that these neurons were located in layer III. With the cryoprobe and cortex at $38^{\circ} \mathrm{C}$, both neurons gave vigorous responses to a visual stimulus. During cooling of the probe to $8^{\circ} \mathrm{C}$ the visually evoked activity was silenced. However, for one neuron some residual, endogenous activity remained. We have seen similar residual, endogenous activity on several occassions. As above, the effects of the cooling are completely reversible.

During cooling of cryoloops to even $0^{\circ} \mathrm{C}$ afferent axons remain active. This observations shows that cooling does not impede action potential transmission in axons and, presumably, the principal effect of the cooling is to slow synaptic mechanisms sufficiently to block synaptic transmission, and the cooling results in inactivation of postsynaptic neurons. In some instances, the silencing of cortical neurons revealed the existence of lower amplitude extracellular action potentials generated by axons, which were previously obscured by the larger potentials generated by cell bodies. Transmission of action potentials in white matter underlying cooled cortex is completely unimpeded by cooling at the pial surface. 


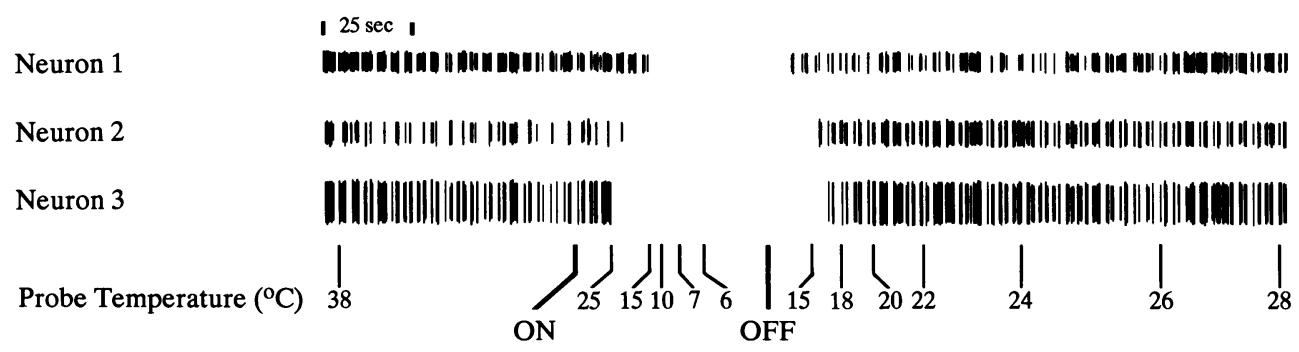

Fig. 6. Action potentials of three neurons before, during, and after cooling of the cortex adjacent to the probe. Before cooling, action potentials were generated to short bars moving to-and-fro across the receptive fields of the neurons. The bursts are synchronously linked to the to-and-fro motion of the stimulus. When the probe was switched on, the numbers of evoked action potentials diminished until no activity was evoked; at this point the neurons in MS cortex were silent. When the probe was switched off, neuronal activity rapidly returned and reached control levels within about $1 \mathrm{~min}$. Reproduced from Lomber et al. (1994) with permission.

\section{Extent of deactivated cortex}

Electrophysiological, thermal and metabolic measures may be used to obtain a reasonable estimate of the extent of deactivated cortex. We have used all three approaches, and each has its own advantages and disadvantages.

\subsection{Electrophysiological measures}

As shown above, extracellular electrophysiological recording methods provide the most direct and accurate measure of neuron deactivation during cooling and, superficially, it is very attractive for assessing the extent of deactivated cortex. However, there are two major factors mitigating its usefulness on a routine basis: (1) A map of evoked and silenced activity at multiple loci with its many cooling cycles is laborious and time consuming to generate. (2) The large number of electrode penetrations necessary to generate a sufficiently detailed map of the deactivated cortex, tends to macerate the tissue and make it unuseable for verifying the integrity of cooled cortex post mortem. Moreover, there is a dependency on evoked activity because some neurons and all afferent axons maintain some level of activity when cooled (Fig. 7). For the latter, the activity may be stimulus linked and may be considerable, but can be ascribed to fibers based on the low amplitude, short duration action potentials (Humphrey, 1979). In the absence of close scrutiny, the afferent fiber activity may be mistaken for neuronal activity and lead to difficulty ascertaining whether a region is silenced or not. Failure to attend to the latter two points leads to a severe under estimation of the extent of deactivated cortex.

\subsection{Thermal measures}

Thermal measurements also provide accurate estimates of extent of deactivated cortex. However, accurate estimates are critically dependent upon knowledge of the temperature below which evoked neural activity is silenced. In cat cerebral cortex and superior colliculus we have used microthermocouples (Omega Engineering ${ }^{\circledR}$, HYP-0-33-1-T-G-60-SMP-M) positioned adjacent to tungsten electrodes to identify $20^{\circ} \mathrm{C}$ as the critical temperature below which neurons fail to be activated by afferent signals. Surprisingly, neuropil temperatures above $24^{\circ} \mathrm{C}$ showed little diminution in evoked activity (Fig. 8), there being a very steep gradient between 24 and $20^{\circ} \mathrm{C}$ along which the number of neuronal action potentials progressively decreases even for vigorously active neurons. These values conform with previous measurements of Jasper et al. (1970) and Benita and Conde (1972).

As with electrophysiological procedures, multiple closely-spaced measurements are required for the most accurate estimates of deactivated cortex. We have used both single and custom-made arrays of microthermocouples to measure temperatures and then, following histological reconstruction of microthermocouple tracks, assigned temperatures to loci along the tracks, and interpolated the course of the $20^{\circ} \mathrm{C}$ thermocline. Neurons at positions between the $20^{\circ} \mathrm{C}$ thermocline and the cryoloop are silenced by the cooling, whereas neurons at more distant positions remain active. As can be seen in Fig. 9, the effect of the cooling is extremely restricted in extent being confined to $1.5-2.5 \mathrm{~mm}$ distance from the cryoloop even when cryoloop temperature is reduced to $1^{\circ} \mathrm{C}$.

Even though temperature measurements are useful and are easier to make than electrophysiological measurements, there can be considerable tedium in taking large numbers of measurements if coolings and reversals are made for each cortical locus measured. As with electrophysiological methods, a dense, accurate assessment of deactivated cortex makes cortical tissue unuseable for histological inspection and verification of structural integrity. However, in many instances, a spacing of $1 \mathrm{~mm}$ between microthermocouple tracks gives sufficient resolution to obtain a reasonably accurate map of the deactivated region with moderate to good tissue preservation between tracks. 
$38^{\circ}$

Neuron

1
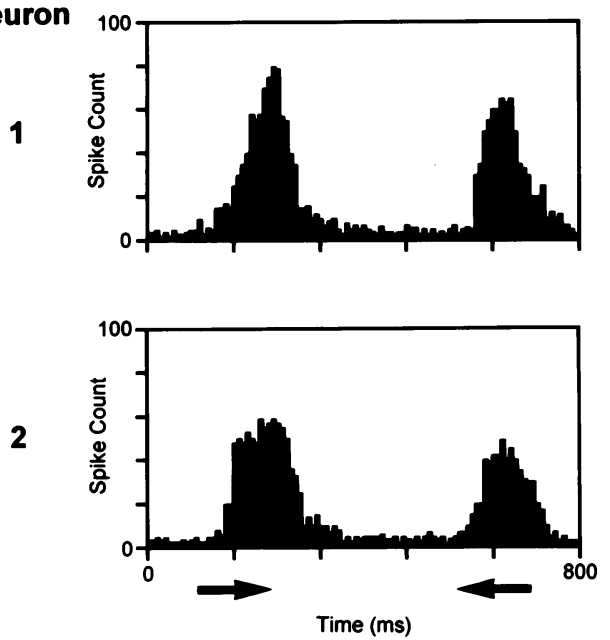

$8^{\circ}$
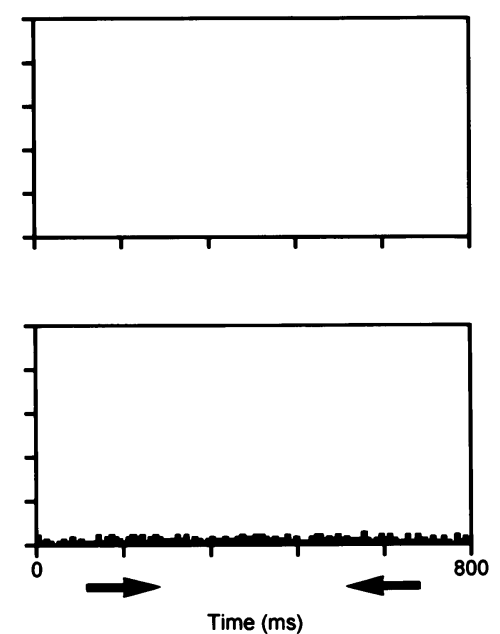

$38^{\circ}$
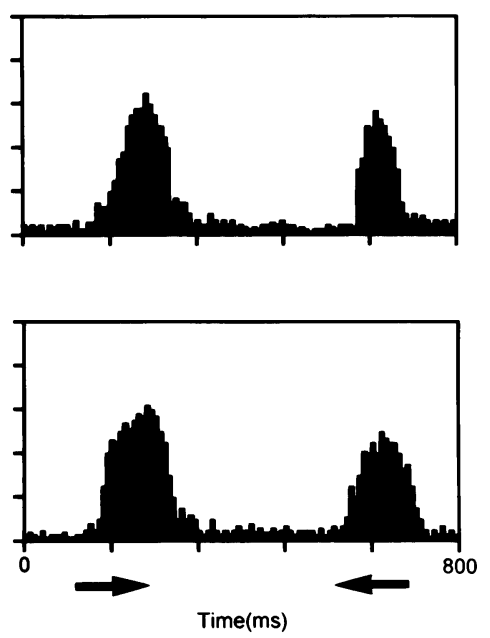

Fig. 7. Histograms of visually-evoked neural activity from two neurons recorded simultaneously in layer III of posterior-middle suprasylvian visual cortex of the cat before (left column), during (middle column) and after (right column) cooling of the probe to $8^{\circ} \mathrm{C}$. Activity fully returned within about $5 \mathrm{~min}$ following the termination of cooling. Arrows indicate relative directions of stimulus movement across the aggregate receptive field.

Thermal measures have other uses. They can provide a measure of the rate of spread of cooling through cortex. For example, Fig. 10, which is based on monkey cortex data shows cortex starts to cool with a delay of only some tens of seconds after a cooling pump is switched on. Much of this delay is accounted for by the time required for the chilled methanol to be transmitted from the methanol/dry ice bath to the cryoloop, as well as surmounting the inertial resistance to cooling by the constant in-flow of warm blood into the target tissue. Regardless of the basis for the delay, cortical tissue

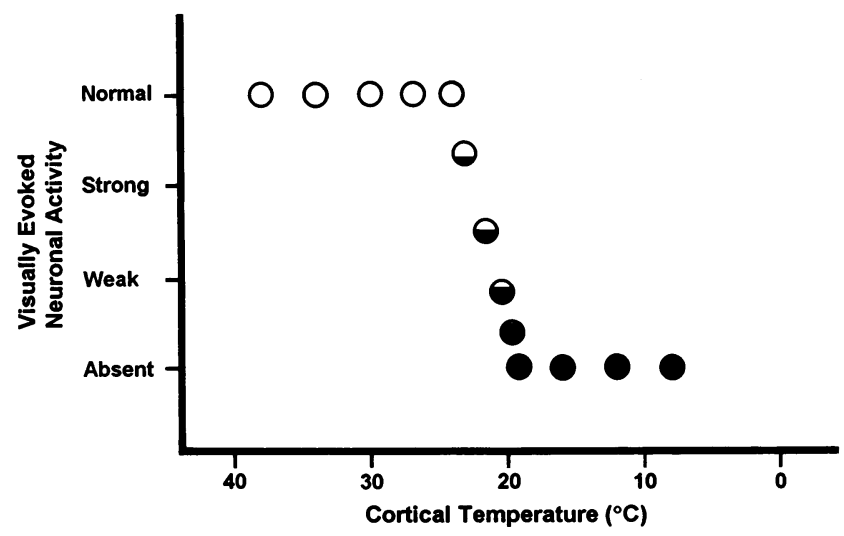

Fig. 8. Relationship of temperature measurements to visually evoked neuronal activity. Recordings were made at four strongly visually driven sites in middle suprasylvian visual cortex of the cat while the cooling probe was turned either on or off. We used a 4-point scale and classified visually-evoked neuronal activity as normal, strong, weak or absent. At recording sites above $24^{\circ} \mathrm{C}$, visually evoked activity was strong and indistinguishable from that evoked at normal body temperature (open circles). At recording site temperatures between 20 and $24^{\circ} \mathrm{C}$, responses weakened in a graded fashion (partially filled circles) until all visually driven activity was completely abolished at a cortical temperature of $20^{\circ} \mathrm{C}$ or less (filled circles). adjacent to the cryoloop reaches a deactivating temperature of $20^{\circ} \mathrm{C}$ within $10 \mathrm{~s}$. However, for deep cortical layers, at a depth of $1.3 \mathrm{~mm}$, the deactivating temperature lags by approximately 1-2 min. Overall, in our hands cortical thickness approximates the limit of cooling deactivation accomplished with the cryoloop. Positions only $2 \mathrm{~mm}$ distance from the cryoloop are cooled only slightly, and positions $5.6 \mathrm{~mm}$ distance are completely unaffected by the cooling. Thus, cooling effects are highly localised to the immediate $1-2 \mathrm{~mm}$ vicinity of the cryoloop.

After the initial delay, as temperature stabilizes, cooling influences remain constant. At the termination of cooling, and cessation of chilled methanol circulation, temperature climbs very rapidly. However, 2-3 minutes is required for temperatures at all distances from the cryloop to return to normal body temperature. For the cryoloop, the lag is largely accounted for by the thermal inertia of the inert cryoloop, which is not actively rewarmed by the immediate in flow of warm blood, as is the case for the neuropil.

Knowledge of arterial vasculature is critical to the accurate design of a deactivation experiment, because arterial patterns contribute greatly to the region of cortex deactivated. Contrary to popular belief, cooling effects in cortex are spread actively by chilled blood and not passively; they bear little or no resemblance to temperature gradients measured in inert materials such as agar or fixed brain tissue.

Clear assymmetries in cooling effects have been identified. For example, cooling probes inserted into the middle suprasylvian sulcus of cat lie across branches of the middle cerebral artery (Fig. 9). With this configuration the cooling is exported further medially than later- 

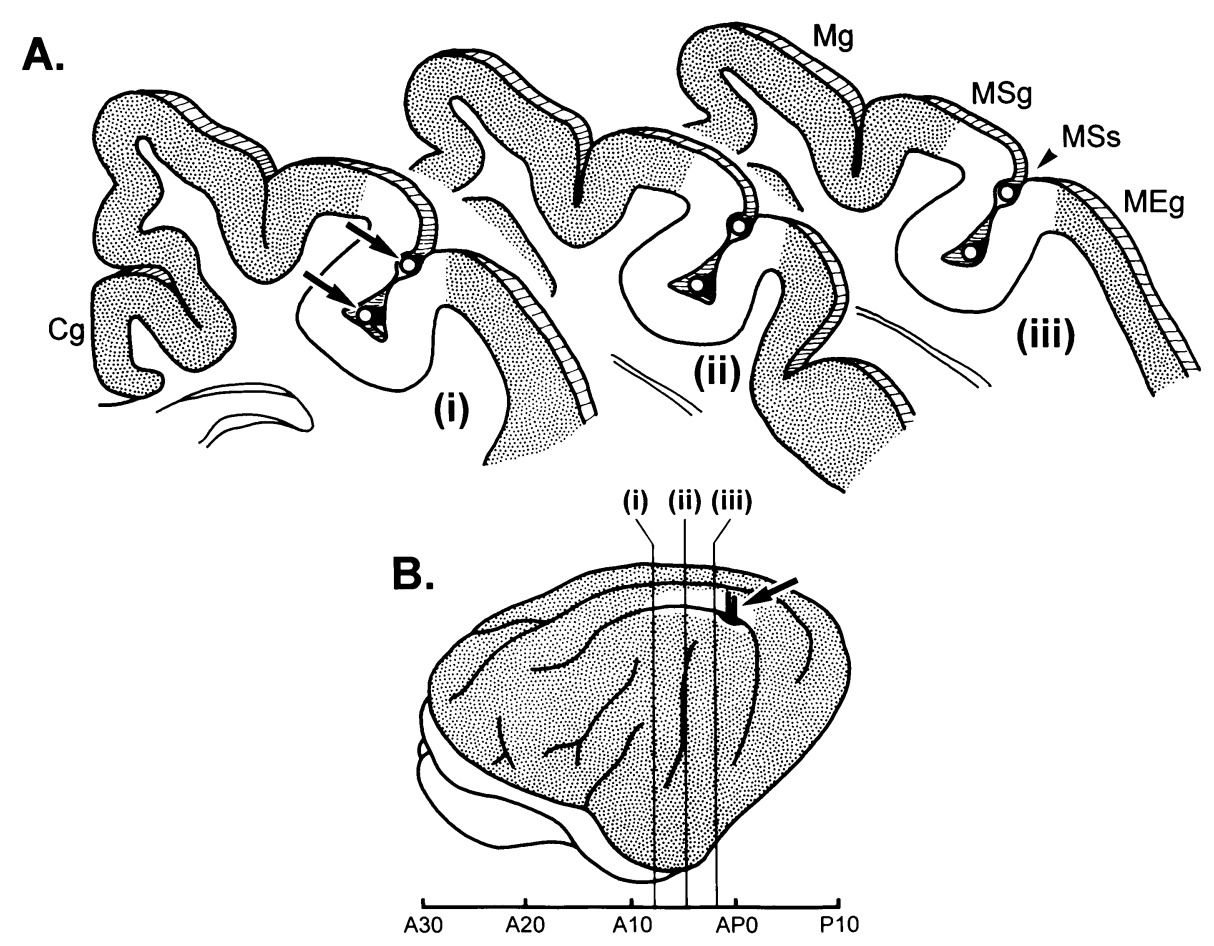

Fig. 9. Position of a cryoloop in the posterior middle suprasylvian sulcus (MSs) and the extent of the deactivation that results when the cryoloop is cooled to $1^{\circ} \mathrm{C}$. Coronal sections (A) show how the cryoloop was placed in the sulcus in contact with both the medial and lateral banks and the resulting deactivated region (white, $<20^{\circ} \mathrm{C}$ ). (B) Lateral view of the left hemisphere to show the site where the cooling tubes exited the sulcus, the extent of the deactivation on the external surface (white), and position of the three coronal sections (i-iii). Note that the deactivation extends further medial than lateral (white). Mg, marginal gyrus; MSg, middle suprasylvian gyrus; MEg, middle ectosylvian gyrus. Arrows indicate the position of the cryoloop and tubes.

ally. In the lateral direction, the spread of cooling is contained within $1.5 \mathrm{~mm}$ of the cryloop by the constant opposition of cooling by warm blood delivered to the region by proximal arterial branches (relative to the heart). In comparison, in the medial direction the spread of cooling is facilitated by the normal flow of blood away from the cryoloop and it extends for a distance of upto $2.5 \mathrm{~mm}$. These estimates are confirmed by measures of reduced uptake of 2DG (Payne and Lomber, 1999)

\subsection{2-Deoxyglucose measures}

A most convenient way to estimate the extent of deactivated cortex is to administer 2-deoxyglucose. The only drawbacks with the method are the expense of the 2DG if a large animal is used, and the exercising of precautions in handling radioactive compounds. However, the technique does have substantial advantages. It provides excellent measures of neural activity levels with considerable anatomical resolution, and it provides both on a global scale. Moreover, normal structure is preserved, and permits visual inspection of tissue for signs of surgical or cooling-induced pathology. These features offer considerable advantages over electrophysiological and temperature assessments of the extent of deactivated cortex.
Deoxyglucose is a direct marker of metabolic activity, and an indirect marker of neural activity (Sokoloff, 1981a,b). The premise of 2DG use in the present application is based on the observation that highly metabolically-active neurons consume more energy and take up more glucose than less active neurons. Since cooling silences evoked responses by neurons and depresses metabolic activity several fold, little 2DG is taken up by the silenced neurons compared to regions at normal temperature and with normal levels of high activity. Consequently, the deactivated region is identified as a very pale region surrounded by dark gray, active tissue in radiograms (Fig. 1 of Payne et al., 1996b; Fig. 1 of Payne and Lomber, 1999). In accord with temperature measurements, 2DG radiograms frequently reveal deactivated regions positioned asymmetrically about the cryoloop (Fig. 9). The dimensions of the asymmetry are similar to those revealed by temperature measurements, and reflect poistion of the cryoloop relative to the underlying arterial vasculature.

In addition to defining the local, cooling impact zone, the cooling modulates 2DG uptake at distant sites in receipt of efferent projections from the cooled cortex (Vanduffel et al., 1997). Comparisons with pathway tracer studies show that the magnitude of the $2 \mathrm{DG}$ modulation is not related linearly to the anatomical 


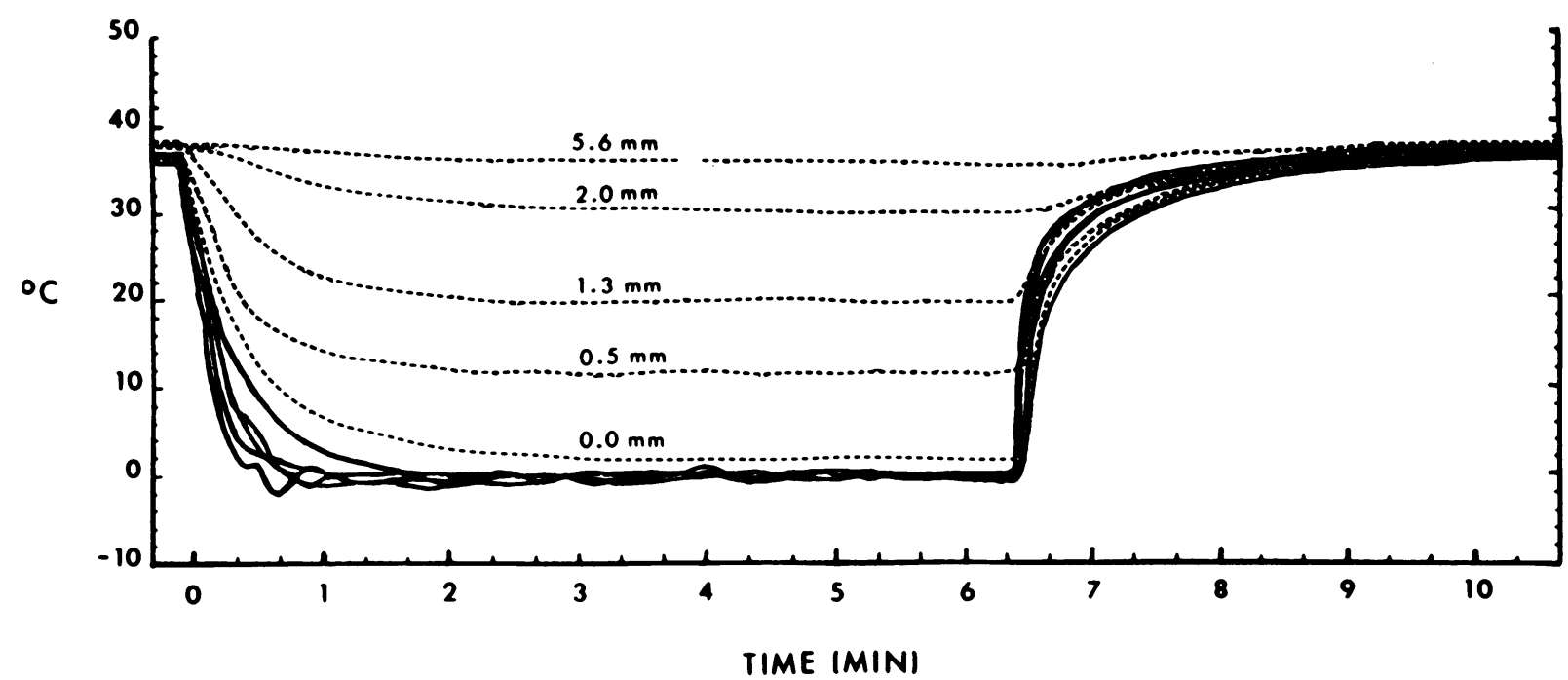

Fig. 10. Time to cool cortical tissue at different distances. Cryoloop temperature is indicated by the solid lines and cortical temperatures at different distances from the cryoloop are indicated by the dashed lines.

strength of connection, but appears to provide a measure of the functional impact of efferent projections (Vanduffel et al., 1997). This auxiliary use and its relevance to studying the functional impact of connections is describe more fully in Payne and Lomber (1999).

\section{Application of cryoloop method to electro-physiological and behavioral experiments}

\subsection{Applications}

The cryoloop method for deactivating cortical tissue has been demonstrated to be a simple and highly effective method for investigating both neuronal interactions using electrophysiological methods and the contributions cerebral network components (and superior colliculus) make to perception, cognition and sensorimotor operations. In both cats and monkeys, cryoloops have been implanted to repeatedly deactivate regions of cerebral cortex or superior colliculus over long periods of time. They have been used successfully to deactivate surface regions (Horel, 1984, 1992, 1996; Lomber et al., 1996a,b), cortex buried within sulci (Lomber et al., 1994, 1996b, Payne et al., 1996a; Hupé et al., 1998), and superior colliculus (Keating and Gooley, 1988; Lomber and Payne, 1996; Payne et al., 1996a). Moreover, when multiple cryoprobes are implanted sophisticated double, and triple, functional dissociations can be made, and the cooling effects of adjacent cryoprobes can be combined to deactivate large regions of cortex. Overall, the use of multiple cryoprobes provides greater flexibility, strengthens the design of experiments, and minimizes the number of animals used in a study.
The time course of effects on individual neurons and behavior differ. Electrophysiological recordings revealed that neurons are silenced very quickly $(<1 \mathrm{~min})$ after switching the cryoprobe on and that neurons are activated again just as quickly after switching the cryoprobe off. In contrast, behavioral impairments take somewhat longer to appear and disappear when cooling is initiated or terminated. The difference in speed with which cooling effects become manifest for neurons and for behaviors is probably linked to the differences in the circuits affected. For individual neurons, the circuits affected are the afferent inputs and local circuitry. In contrast, impairments in behavior are accomplished by both local silencing of neurons beneath the cryoloop and removal of efferent signals normally transmitted to other parts of the brain. It seems likely that the silencing of a single neuron's activity is simpler and easier to accomplish compared to the abolition of a behavior which requires the silencing of large populations of neurons and the elimination of substantial numbers of efferent signals.

\section{Knowledge derived from cooling deactivations}

\subsection{Complete deficits}

It is important to recognize that the cooling does not automatically reveal the contribution a deactivated region makes to behavior, but rather the capacity of other brain regions to maintain the behavior in the absence of the deactivated region. Little can be inferred when behavioral performance is normal. However, a region can be safely inferred as essential if performance during cooling is reduced to chance levels or eliminated entirely. 


\section{Week}
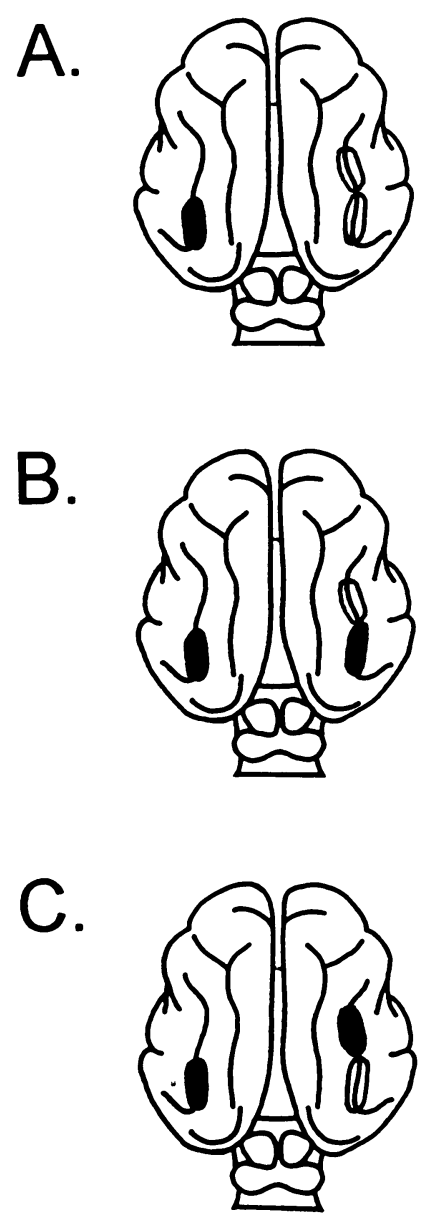
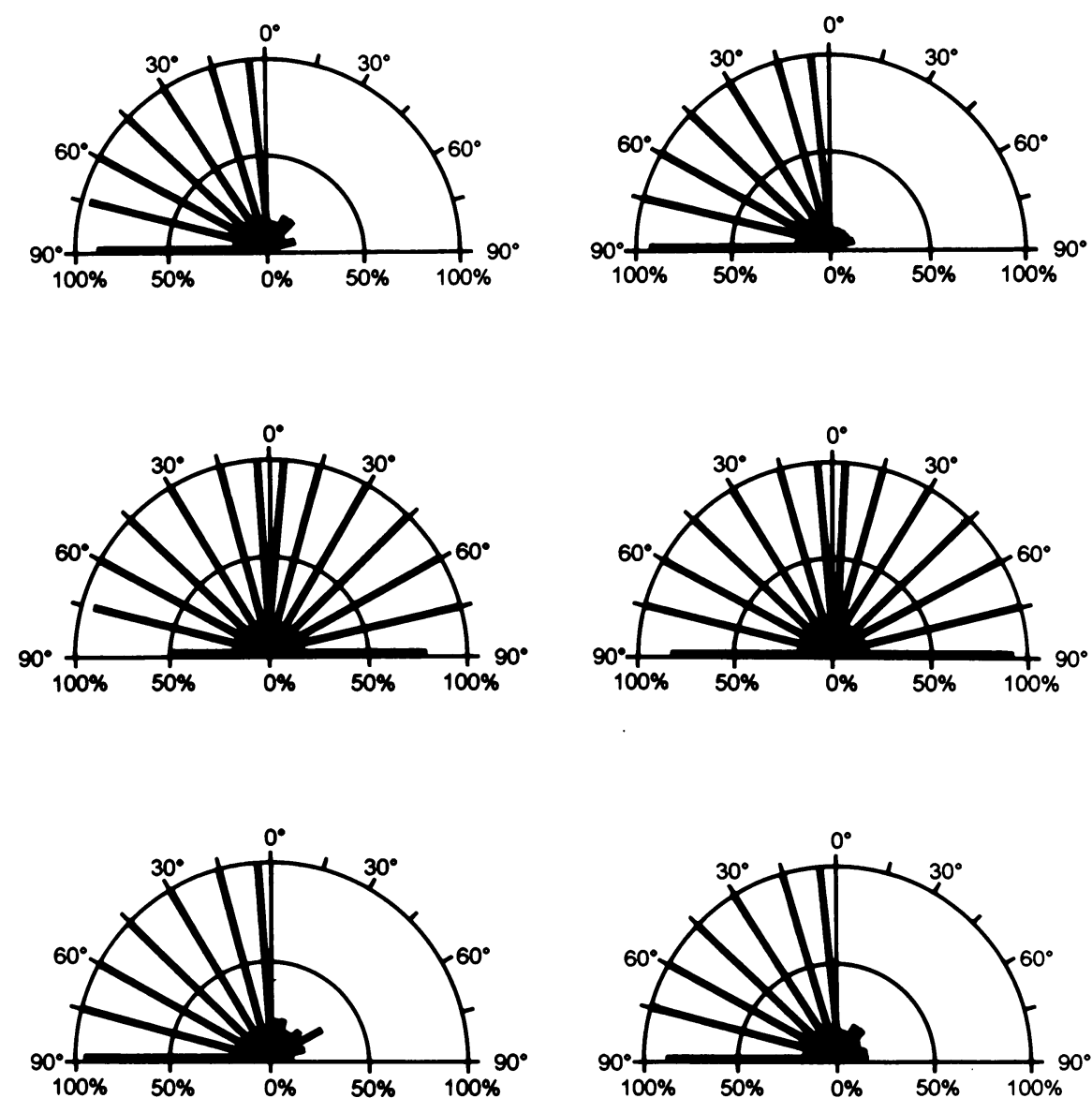

2.75 Years

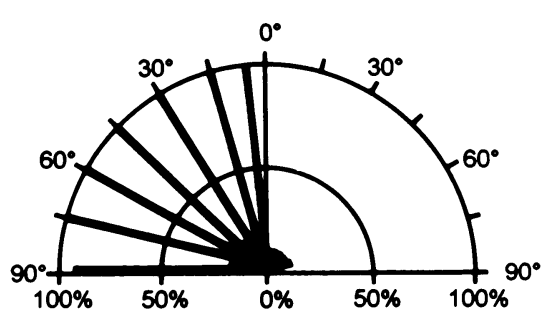

Fig. 11. Visual orienting data from a cat which had cryoloops implanted for nearly 3 years. Left column shows a dorsal view of the cerebrum and the positions of three cryoloops over the cortical surface. Left hemisphere contained one posterior-middle suprasylvian (pMS) sulcal cryoloop. Right hemisphere contained a mirror symmetric pMS cortex cryoloop and an anterior-middle suprasylvian (aMS) sulcal cryoloop. Middle and right columns show behavioral performance within 1 week and following 2.75 years after implantation, respectively. (A) Unilateral deactivation of pMS results in a profound neglect of stimuli introduced into the contracooled visual hemifield (Lomber and Payne, 1996; Payne et al., 1996a). (B) The additional cooling of pMS cortex in the contralateral hemisphere restores visual orienting responses into the previously neglected hemifield (Lomber and Payne, 1996). (C) The additional cooling of an adjacent, non-mirror symmetric, site in the opposite hemisphere, such as aMS does not result in a restoration of orienting responses. The results in all three of these testing conditions were virtually identical regardless of the length of time after implantation (compare middle and right columns).

This point is emphasized by the ability of a cat to detect and orient toward a stimulus during unilateral and bilateral deactivation of posterior middle suprasylvian (pMS) cortex. Unilateral deactivation of pMS cortex induces a neglect of stimuli presented in the hemifield contralateral to the cooling. It might be expected that with bilateral cooling of pMS cortex would result in neglect of stimuli presented anywhere in the visual field. In fact, the exact opposite result is obtained, and the addition of the second pMS cooling in the opposite hemisphere reverses the deficit induced by unilateral cooling (Fig. 11; Lomber and Payne, 1996). The restitution is highly specific to homotopic cortex because operation of a second cooling probe at an asymmetric position in contact with aMS cortex, or other sites, does not reverse the unilateral neglect. Importantly, the deficit induced by unilateral cooling of pMS cortex and its reversal do not attenuate over either time or large numbers of deactivations because they are as strong and as reproducible 3 years after the initial deactivation and reversal was carried out (Fig. 11).

\subsection{Incomplete deficits}

The inferences that can be made when there is an incomplete impairment are more difficult to reach. Partial reduction in performance may occur because of shared processing properties between the deactivated region and another region or incomplete deactivation of one single region. In many instances, the latter can 
be tested by increasing the cooling effect by lowering probe temperature and deactivating a larger volume of cortex or by addition of cooling of an adjacent cryprobe. In either case, an increase in the magnitude of the deficit reveals an initial incomplete block. This is a very useful strategy for maximising the deficits and increasing statistical reliability. Another factor that may lead to incomplete blockade is that the task is not sufficiently difficult. For example, with the maximum size of probes we use, we have been unable to impair discriminations of differences in the direction of motions between two patterns when the difference in direction is $45^{\circ}$ or more. In contrast, differences of $30^{\circ}$ are readily impaired by cooling parts of middle suprasylvian cortex (Lomber et al., 1996a).

\subsection{Neural compensations}

Importantly, under most conditions the cooling method precludes neural compensations, which frequently accompany lesion deactivations. These compensations mask the original lesion-induced deficits (see Payne et al. (1996b) for examples and discussion). The dominant factor distinguishing the lesion and cooling deactivations is likely to be the duration of the deactivation. Following lesions, animals live permanently with the neural defect and there is considerable opportunity for prolonged interactions between the animal with the defect and the environment. These interactions may result in modifications in remaining circuitry that reduce the severity of the handicap. In contrast, these same influences have little time to act on the nervous system during cooling, which occupies only $5-10 \%$ or less of each day, and there is little or no strengthening of secondary circuits. Further, any compensatory changes initiated during brief cumulative cooling periods do not seem to accumulate over repeated cooling sessions, because either the changes are very minor or they are reversed during the much longer intervals when the brain is functioning normally.

Support for the above supposition is provided by evidence showing that under certain circumstances cats are able to learn certain form discriminations while cortex is cooled for prolonged periods. However, this 'compensation' can only be demonstrated for form discriminations in which the discriminanda differ in multiple ways, and if the discrimination engram is firmly established prior to cooling deactivation (Lomber et al., 1996b). In this instance, the initial cooling deficit may be one of access to the engram, and it is this initial access difficulty which is overcome with training during cooling It should not be confused with new learning, per se, of the form discriminations. Regardless of interpretation, these results provide evidence for plasticity in the cerebral network for signals and processing to circumvent the deactivated region.

\subsection{Laminar contributions to behavior}

So far, both lesion and cooling studies have concentrated on deactivating regions in the two dimensional sheet of the cortex. Recent evidence suggests that it may also be profitable to use cooling to attempt to dissect laminar contributions to visually-guided behaviors. For example, cooling of a pMS cryoprobe to $8-9^{\circ} \mathrm{C}$ is sufficient to completely impair discriminations of differences in movement direction of $30^{\circ}$, but has virtually no impact on the ability of a cat to orient to a stimulus appearing in the cat's visual periphery. Performance of this latter task is only abolished when the cryoloop temperature is reduced to $3^{\circ} \mathrm{C}$ (Fig. 12A). Subsequent temperature measurements made when the cryoloop is at $8-9^{\circ} \mathrm{C}$ reveal that the $20^{\circ} \mathrm{C}$ thermocline is positioned at the level of the middle cortical layers, indicating that the superficial layers $1-3$ are deactivated. In contrast, when the cryoloop is cooled to $3^{\circ} \mathrm{C}$, the $20^{\circ} \mathrm{C}$ thermocline is positioned at the level of the gray/white matter interface, indicating that all layers are deactivated (Fig. 12B). The inferences from these results are that the superficial layers contribute importantly to motion discriminations and have little or no effect upon detection of and orienting toward stimuli, which is subserved by neurons located in the deeper layers.

\section{Recommended supplementary procedures in behavioral studies}

Finally, we recommend that during and at the end of the testing phase that periodic and terminal checks on behavior and cooling be carried out. Some of these supplementary procedures can also be applied in electrophysiological studies.

\subsection{Periodic checks}

We recommend that behavioral tests be repeated at intervals to verify constancy of behaviors and coolinginduced deficits over time. A supplementary step can be to implant arrays of microthermocouples into cortex in the vicinity of the cooling probes to verify constancy of cortical cooling across test sessions.

\subsection{Terminal checks}

At the end of behavioral testing, we recommend assigning animals to subgroups to verify cooling induced deactivation using electrophysiological methods or map the deactivated region of cortex with microthermocouples or 2-deoxy- ${ }^{14} \mathrm{C}$-glucose (2DG; Payne and Lomber, 1999). Finally, a critical step in our view is to carry out histological procedures to verify the absence of damage from regions in contact with the cryoprobe. 

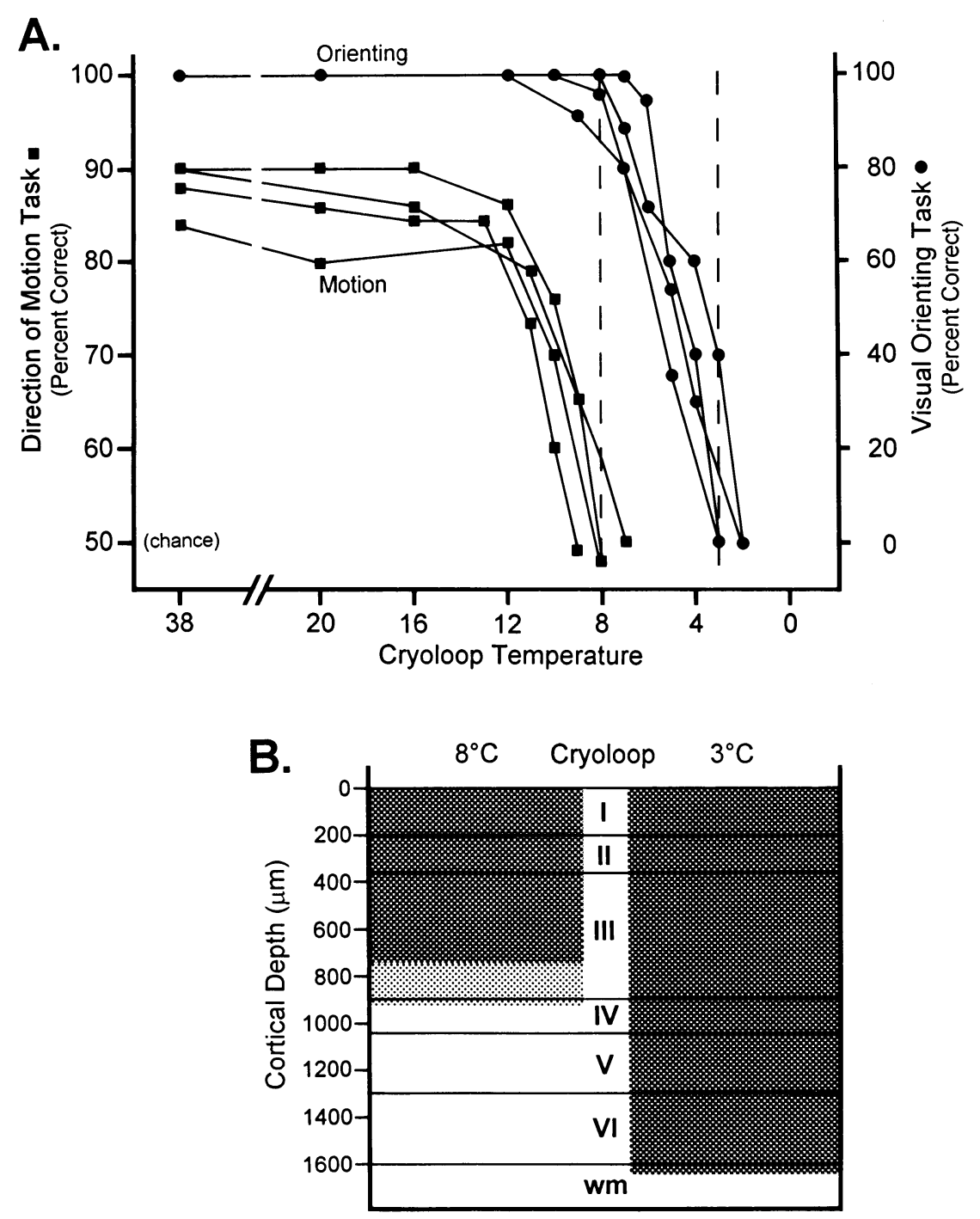

Fig. 12. (A) Behavioral performance from four cats on two tasks during cooling of a posterior-middle suprasylvian (pMS) sulcal cryoloop to different temperatures. While performing a direction of motion task (black squares), no impairment was identified for cryoloop temperatures $\geq 12^{\circ} \mathrm{C}$. However, performance fell to chance levels for all cats when cryoloop temperature dropped to about $8^{\circ} \mathrm{C}$. While performing a visual orienting task (black circles), little or no impairment was identified for cryoloop temperatures $\geq 8^{\circ} \mathrm{C}$. However, performance was completely impaired when cryoloop temperatures dropped to $3^{\circ} \mathrm{C}$. (B) Just prior to sacrifice, the extent of pMS cortex deactivated at cryoloop temperatures of $8^{\circ}$ and $3^{\circ} \mathrm{C}$ was recorded. At a cryoloop temperature of $8^{\circ} \mathrm{C}$, cortical temperatures $<20^{\circ} \mathrm{C}$ were recorded throughout layers I, II and the superficial 2/3's of layer III (dark stipple). Temperatures between 20 and $24^{\circ} \mathrm{C}$ were recorded in deep layer III and superficial layer IV (light stipple). Therefore, layers I and II and superficial III were fully deactivated and deep III and superficial IV were partially deactivated (see Fig. 8). However, at a cryoloop temperature of $3^{\circ} \mathrm{C}$, the full extent of pMS cortex was $<20^{\circ} \mathrm{C}$ and fully deactivated (dark stipple). Therefore, only the superficial layers needed to be deactivated to produce an impairment on the direction of motion task, while the deep layers also had to be deactivated in order to produce an impairment on the visual orienting task.

(1) Electrophysiology: Routine electrophysiological methods (Payne, 1990) are used to verify cooling deactivation of neuronal activity and determine directly the laminar and spatial extent of the cortical area deactivated (e.g. Lomber et al., 1994, 1996b; Payne et al., 1996b). Objective measures of cooling induced deactivation are obtained using Brainwave Systems' 'Discovery' software to collect multiple channels of extracellular spike activity (e.g. Lomber et al., 1994, 1996a; Mendola and Payne, 1993; Payne et al., 1991, 1996b), and calculation of a cooling block: normal ratio (B:N ratio, Malpeli, 1983). When $\mathrm{B}: \mathrm{N}=0$, neurons are silenced during cooling; when $\mathrm{B}: \mathrm{N}<1$, activity is diminished; and when $\mathrm{B}: \mathrm{N}=1$, there is no change in activity during cooling.

(2) Thermoclines: Multiple tracks with micro-thermocouples, single or an array of four, are made to map thermoclines and temperature gradients in the vicinity of the cooled probes. The tracks are reconstructed in histological sections, and positions with temperatures of 
$\leq 20^{\circ} \mathrm{C}$ identified. The envelope enclosing these sites represents the area silenced (e.g. Figs. 7, 8 and 10 in Lomber et al., 1996b; Fig. 11 in Lomber and Payne, 1998).

\section{Conclusion}

The cryoloop cooling deactivation has many advantages over other deactivation techniques. It is adaptable to both the size and shape of the brain region to be deactivated, it requires only a modest cost and it is easy to maintain. Furthermore, the system can be used in large animals such as monkeys and cats, and it can be adapted for use in small animals such as rats and hamsters.

\section{Acknowledgements}

We thank Dorothy Pytko-Joiner for assistance, and the National Institute of Neurological and Disorders and Stroke for financial support.

\section{References}

Alexander GE, Goldman PS. Functional development of the dosolateral prefrontal cortex: an analysis utilizing reversible cryogenic depression. Brain Res 1978;143:233-49.

Benita M, Conde H. Effects of cooling upon conduction and synaptic transmission. Brain Res 1972;36:133-51.

Campeau S, Davis M. Reversible neural inactivation by cooling in anesthetized and freely behaving rats. J Neurosci Methods 1990;32:25-35.

Dondey M, Albe-Fessard D, Le Beau J. Premières applications neurophysiologiques d'une méthode permettant le blocage électif et réversible de structures centrales par réfrigération localisée. Electroenceph Clin Neurophsyiol 1962;14:758-63.

Fuster JM, Alexander GE. Delayed response deficit by cryogenic depression of frontal cortex. Brain Res 1970;81:393-400.

Fuster JM, Bauer RH. Visual short-term memory deficit from hypothermia of frontal cortex. Brain Res 1974;20:85-90.

Girard P, Bullier J. Visual activity in area V2 during reversible inactivation of area 17 in te macaue monkey. J Neurophysiol 1989;62:1287-302.

Girard P, Salin P-A, Bullier J. Visual activity in macaque area V4 depends on area 17 input. NeuroReport 1991a;2:81-4.

Girard P, Salin P-A, Bullier J. Visual activity in areas V3a and V3 during reversible inactivation of area $\mathrm{V} 1$ in the macaque monkey. J Neurophysiol 1991b;66:1493-503.

Girard P, Salin P-A, Bullier J. Response selectivity of neurons in area MT of the macaque monkey during reversible inactivation of area V1. J Neurophysiol 1992;67:1437-46.

Girard P, Lomber SG, Payne BR, Coquard B, Bullier J. Deficits in form perception during reversible inactivation of macaque area V4. Soc Neurosci Abstracts, 23 1997;2:2062.

Goldman PS, Alexander GE. Maturation of prefrontal cortex in the monkey revealed by local reversible cryogenic depression. Nature 1977;267:613-5.
Horel JA. Cold lesions in inferotemporal cortex produce reversible deficits in learning and retention of visual discriminations. Physiol Psychol 1984;12:259-70.

Horel JA. Use of cold to reversibly suppress local brain function in behaving animals. In: Conn PM, editor. Methods in Neurosciences: Lesions and Transplantation, vol. 7. New York: Academic Press, 1991:97-110.

Horel JA. Retrieval of active and inactive visual discriminations while temporal cortex is suppressed with cold. Behav. Brain Res 1992;51:193-201.

Horel JA. Perception, learning and identification studied with reversible suppression of cortical visual areas in monkeys. Behav. Brain Res. 1996;76:199-214.

Horel JA, Pytko-Joiner DE, Voytko ML, Salsbury K. The performance of visual tasks while segments of inferotemporal cortex are supressed by cold. Behav Brain Res 1987;23:29-42.

Horel JA, Voytko ML, Salsbury KG. Visual learning suppressed by cooling the temporal pole. Behav Neurosci 1984;98:310-24.

Humphrey DR. Electrophysiological Techniques. Bethesda, MD: Society for Neuroscience, 1979:199-259.

Hupé JM, James AC, Payne BR, Lomber SG, Girard P, Bullier J. Cortical feedback improves discrimination between figure and ground by V1, V2 and V3 neurons. Nature 1998;394:784-87.

Jasper H, Shacter DG, Montplaisir J. The effect of local cooling upon spontaneous and evoked electrical activity of cerebral cortex. Can J Physiol Pharmacol 1970;48:640-52.

Keating EG, Gooley SG. Saccadic disorders caused by cooling the superior colliculus or the frontal eye fields, or from combined lesions of both structures. Brain Res 1988;438:247-55.

Lomber SG, Payne BR. Removal of two halves restores the whole: Reversal of visual hemineglect during bilateral cortical or collicular inactivation in the cat. Vis Neurosci 1996;13:1143-56.

Lomber SG, Payne BR. Evidence for differential laminar contributions to visually-guided behaviors revealed by localized cerebral cooling deactivation. Cereb Cortex 1998 (in press).

Lomber SG, Cornwell P, Sun JS, MacNeil MA, Payne BR. Reversible inactivation of visual processing operations in middle suprasylvian cortex of the behaving cat. Proc Natl Acad Sci (USA) 1994;91:2999-3003.

Lomber SG, Payne BR, Cornwell P. Learning and recall of form discriminations during reversible cooling deactivation of ventralposterior suprasylvian cortex in the cat. Proc Natl Acad Sci (USA) 1996a;93:1654-8.

Lomber SG, Payne BR, Cornwell P, Long KD. Perceptual and cognitive visual functions of parietal and temporal cortices in the cat. Cereb Cortex 1996b;6:673-95.

Malpeli JG. Activity of cells in area 17 of the cat in absence of input from layer A of the lateral geniculate nucleus. J Neurophysiol 1983;49:595-610.

Michalski A, Wimborne BM, Henry GH. The effect of reversible cooling of cat's primary visual cortex on the responses of area 21a neurons. J Physiol (London) 1993;466:133-56.

Michalski A, Wimborne BM, Henry GH. The role of ipsilateral and contralateral inputs from primary cortex in responses of area 21a neurons in cats. Vis Neurosci 1994;11:839-49.

Mendola JD, Payne BR. Direction selectivity and physiological compensation in the superior colliculus following removal of areas 17 and 18. Vis Neurosci 1993;10:1019-26.

Olson CR, Lawler K. Cortical and subcortical afferent connections of a posterior division of feline area 7 (area $7 \mathrm{p}$ ). J Comp Neurol 1987;259:13-30.

Pasternak T, Merrigan WH. Motion perception following lesions of the superior temporal sulcus in the monkey. Cereb Cortex 1994;4:247-59. 
Payne BR. The representation of the ipsilateral visual field in the transition zone between areas 17 and 18 of the cat's cerebral cortex. Vis Neurosci 1990;4:445-74.

Payne BR, Lomber SG. A method to assess the functional impact of cerebral connections on target populations of neurons. J Neurosci Methods 1999;86:195-208.

Payne BR, Lomber SG, Villa AE. Lesion-induced network plasticity in remote brain areas. Trends Neurosci 1997;20:348-9.

Payne BR, Siwek DF, Lomber SG. Complex transcallosal interactions in visual cortex. Vis Neurosci 1991;3:283-9.

Payne BR, Lomber SG, Geeraerts S, van der Gucht E, Vandenbussche E. Reversible visual hemineglect. Proc Natl Acad Sci (USA) 1996a;93:290-4.

Payne BR, Lomber SG, Villa AE, Bullier J. Reversible inactivation of cortical network components. Trends Neurosci 1996b;19:535-42.

Salsbury KG, Horel JA. A cryogenic implant for producing reversible functional brain lesions. Behav Res Methods Instrum 1983;15:433-6.

Sandell J, Schiller PH. Effect of cooling area 18 on striate cortex cells in the squirrel monkey. J Neurophysiol 1982;48:38-48.

Schiller PH, Malpeli JG. The effect of striate cooling on area 18 cells in the monkey. Brain Res 1977;126:366-9.

Sherk H. Area 18 cell responses in cat during reversible inactivation of area 17. J Neurophysiol 1978;41:204-15.
Shindy WW, Posley KA, Fuster JA. Reversible deficit in haptic delay tasks from cooling prefrontal cortex. Cereb Cortex 1994;4:44350.

Skinner JE, Lindsley DB. Reversible cryogenic blockade of neural function in the brain of unrestrained animals. Science 1968;161:595-7.

Sokoloff L. The deoxyglucose method for the measurement of local glucose utilization and the mapping of local functional activity in the central nervous system. Int Rev Neurobiol 1981a;22:287-333.

Sokoloff L. The F.O. Schmitt Lecture in Neuroscience 1980. The relationship between function and energy metabolism: its use in the localization of functional activity in the nervous system. Neurosci Res Prog Bull 1981b;19:159-207.

Tusa RJ, Palmer LA. Retinotopic organization of areas 20 and 21 in the cat. J Comp Neurol 1980;193:147-64.

Vanduffel W, Payne BR, Lomber SG, Orban GA. Functional impact of cerebral connections. Proc Natl Acad Sci (USA) 1997;94:761720.

Yamasaki DS, Wurtz RH. Recovery of function after lesions in the superior temporal sulcus in the monkey. J Neurophysiol 1991;66:651-73.

Zhang J, Ni H, Harper RM. A miniaturized cryoprobe for functional neuronal blockade in freely moving animals. J Neurosci Methods 1986;16:79-87. 